\title{
Influências de Atta spp. (Hymenoptera: Formicidae) na recuperação da vegetação pós-fogo em floresta de transição amazônica
}

\author{
Karine Santana CARVALHO ${ }^{1}$, Jennifer BALCH², Paulo MOUTINHO ${ }^{3}$
}

\begin{abstract}
RESUMO
Este estudo investigou o papel das saúvas na recuperação da vegetação pós-fogo. Foi hipotetizado que a perturbação do fogo aumenta a abundância de ninhos de saúva (1) e as atividades de remoção de sementes (2) e desfolha (3), especialmente no ambiente de borda. Os ninhos de saúva foram inventariados e mapeados e após 17 meses, checados para o registro de sua atividade e de novas colônias emergindo na área. Realizou-se experimentos comparativos de remoção de sementes e desfolha de plântulas em duas parcelas de 50 ha, uma submetida a fogo anual (tratamento) e outra sem fogo (controle). A abundância de ninhos de saúva foi maior na parcela tratamento que na parcela controle, especialmente na borda da floresta. As espécies encontradas foram: Atta cephalotes, A. laevigata e A. sexdens, sendo esta última a espécie mais abundante e que mostrou aumento em número de colônias ativas após 17 meses. $\mathrm{O}$ aumento na abundância de ninhos na parcela tratamento foi acompanhado por um aumento na atividade dessas formigas. Enquanto mais de duas folhas foram arrancadas por plântula nessa parcela, menos de uma foi registrada na parcela controle. Também a abundância média de galhos desfolhados e de sementes removidas por saúvas foi maior na parcela tratamento que na parcela controle. As saúvas tanto podem diminuir ou retardar o processo de regeneração florestal pós-fogo, quanto acelerá-lo, devido à elevada predação seletiva que favorece as espécies menos palatáveis. Desta forma, suas atividades podem modificador apenas a composição da vegetação recuperando-se do fogo.
\end{abstract}

PALAVRAS-CHAVE: saúvas, herbivoria, remoção de sementes, regeneração florestal.

\section{Influences of leafcutter ant Atta spp. (Hymenoptera: Formicidae) on vegetation recovery after fire in Amazonian transitional forest}

\begin{abstract}
In this study we investigated the role of leaf-cutting ants in the post-fire vegetation recovery. We hypothesized that a forest plot submitted to annual fire presents: (1) higher abundance of leaf-cutting ant nests; (2) higher removal of seeds; and (3) higher herbivory rates of leaf-cutting ants, when compared to the forest plots without fire (control). The leaf-cutting ant nests were sampled, mapped, and checked up (after 17 months) to register their activity and new colonies emerging from the plot. We made comparative experiments of seeds removal and seedlings herbivory in two 50 ha plots, one submitted to annual fire and another without fire. The abundance of leaf-cutting nests was higher in the plot submitted to fire than in the control plot. The species found were: Atta cephalotes, A. laevigata, and A. sexdens, being the latter the most abundant and the one that showed an increase of active colonies after 17 months. The plot submitted to fire showed a higher abundance of seeds removed by leafcutting ants than the control. While more than two leaves were ripped per seedling in the plot under fire, less than one was registered in the control plot. It was also observed that the average abundance of seedlings attacked by leaf-cutting ant in the fire plot was higher than in the plot without fire. This study shows that the leaf-cutting ants may reduce seed germination and seedling recruitment, affecting the reproductive success and the composition of vegetation recovering from fire. Alternatively, the leaf-cutting ants may also speed up the regeneration process of unpalatable plant species. In this sense, we may conclude that the presence of leaf-cutting may interfere in the species composition of post fire forest.
\end{abstract}

KEY-WORDS: leaf-cutting ants, herbivory, seed removal, forest regeneration.

\footnotetext{
1 Universidade Estadual do Sudoeste da Bahia. E-mail: ksczool@yahoo.com.br

2 School of Forestry and Environmental Studies, Yale University. E-mail: balch@nceas.ucsb.edu

${ }^{3}$ Instituto de Pesquisa Ambiental da Amazônia. E-mail: moutinho.ipam.org
} 


\section{INTRODUÇÃO}

O fogo é utilizado como ferramenta de manejo de ecossistemas desde a era Mesolítica (Moore 1996). No entanto, como agente de perturbação, é um evento infreqüente na floresta amazônica (Uhl e Kauffman 1990; Meggers 1994; Nepstad et al. 1996; Saldarriaga et al. 1998; Barlow e Peres 2003) e os seus efeitos na dinâmica e processos florestais ainda são pouco entendidos (Moore 1996; Cochrane et al. 1999).

Embora infreqüentes, os incêndios florestais têm aumentado bastante nas últimas décadas por conta do padrão de uso da terra na região e mudanças nos regimes climáticos em função do aquecimento global (Cochrane et al. 1999; Nepstad et al. 1999; Curran et al. 1999; Siegert et al. 2001; Cochrane e Laurance, 2002). Desta forma, as plantas evolutivamente não adaptadas a esse tipo de perturbação (Uhl e Kauffman 1990; Cochrane et al. 1999; Nepstad et al. 1999) sofrem altas taxas de mortalidade que pode chegar a 50\% das árvores (Barlow et al. 2003; Haugaasen et al. 2003).

Os insetos também podem experimentar imediata redução de suas populaçôes, à exceção de algumas espécies que vivem abaixo da superfície do solo (Araújo et al. 2004). As saúvas (formiga cortadeira do gênero $A t t a$ ), por exemplo, constroem ninhos profundos e com arquitetura estabilizadora de flutuaçôes de temperatura e umidade (Roces e Kleineidam 2000; Farji-Brener 2000).

Além de resilientes ao fogo, as saúvas proliferam em hábitats alterados (Rao 2000; Santos et al. 2008) ou em estágios iniciais de sucessão, quando há maior disponibilidade de luz no ambiente e uma predominância de plantas dependentes deste recurso (Vasconcelos e Cherrett 1997). Desta forma, a densidade de ninhos aumenta em função do aumento da perturbação florestal, podendo ser até 30 vezes maior em floresta secundária quando comparada com floresta primária (Vasconcelos e Cherrett 1995). Assim, os ninhos estabelecidos em áreas de borda, desenvolvem-se melhor do que em locais sombreados (Barbosa et al. 2005; Vasconcelos et al. 2006; Urbas et al. 2007; Wirth et al. 2007; Meyer et al. 2009). O ambiente de borda é dominado por plantas pioneiras que investem mais em crescimento que em defesas contra herbívoros (Fowler 1983; Jaffé e Vilela 1989, Vasconcelos e Cherrettt 1995; Farji-Brenner 2001).

Em um cenário de intervençóes antropogênicas alterando radicalmente as paisagens amazônicas, as saúvas podem influenciar a estrutura da vegetação que se estabelece (FarjiBrener e Silva 1995; Garrettson et al. 1998; Farji-Brener e Illes 2000; Farji-Brener e Medina 2000; Farji-Brener 2005) tanto por meio de sua herbivoria, quanto por sua atividade de remoção de sementes (Moutinho 1995, 1998; Vasconcelos e Cherrett 1998). O ataque das formigas a folhas $(12-17 \% \mathrm{da}$ produção anual de uma floresta tropical) ramos e até mesmo casca das árvores, têm um efeito direto na produtividade primária da vegetação pela perda de biomassa (Buckley 1982; Majer 1989).

Neste contexto, o objetivo deste estudo foi investigar o papel das saúvas na recuperação da vegetação após evento de incêndio. Especificamente, foi testado se a perturbação do fogo aumentou a abundância de ninhos de saúva (1) e as atividades de remoçáo de sementes (2) e desfolha (3), especialmente no ambiente de borda.

\section{MATERIAL E MÉTODOS}

A área de estudo está localizada na fazenda Tanguro, município de Querência $\left(13.04^{\circ} \mathrm{S}, 52.23^{\circ} \mathrm{W}\right)$, Mato Grosso, Brasil. Esta área está inserida no ecótono Cerrado-Amazônia e na ecorregião Florestas Secas do Mato Grosso (Oren 2005). A temperatura média anual da região é $23,5^{\circ} \mathrm{C}$ com pluviosidade variando entre 1.800 a $2.000 \mathrm{~mm}$. Ocorre uma estação seca entre maio e setembro e uma estaçáo chuvosa entre outubro e abril. Os solos são do tipo latossolo amarelo (Balch et al. 2008).

Este estudo faz parte do Projeto Savanizaçáo liderado pelo Instituto de Pesquisa Ambiental da Amazônia (IPAM) que visa investigar se os efeitos dos incêndios florestais recorrentes afetam a susceptibilidade da floresta a futuros incêndios e induzem a uma "savanização" da vegetaçáo. O projeto abrange áreas de $150 \mathrm{ha}$, compostas de parcelas de 50 ha submetidas a queimas experimentais periódicas (anual e tri-anual) e uma parcela controle, onde não há queima. Os incêndios florestais experimentais estáo sendo realizados desde agosto de 2004.

Foram selecionadas duas parcelas de 50 ha: uma perturbada anualmente por fogo (parcela tratamento) e outra sem essa perturbação (parcela controle). Os incêndios ocorreram entre 9:00 e 16:00 h durante 3-4 dias consecutivos em agosto de 2004 e setembro de 2005. O fogo, ateado manualmente com tochas de querosene, é do tipo rasteiro, ou seja, aquele que queima apenas serapilheira e não atinge árvores em pé (para mais detalhes veja Balch et al. 2008).

Para verificar se a abundância de ninhos de saúvas é maior na parcela tratamento do que na parcela controle, foram realizados dois inventários dos ninhos de saúva. O primeiro foi realizado em fevereiro de 2005 e o segundo em julho de 2007. No primeiro inventário, foram estabelecidos 20 transectos (10 transectos por parcela), cada uma com um km de extensão e 40 $\mathrm{m}$ de largura utilizando-se trilhas já existentes na área. Todos os ninhos ativos saúva avistados dentro destas transecçôes foram registrados e mapeados. A disposição espacial dos ninhos em cada parcela foi utilizada para informaçóes sua distância em relação à borda da floresta.

A atividade dos ninhos foi classificada como a resposta positiva das formigas aos seguintes estímulos: introdução de uma mangueira nos olheiros, seguido de assopro (por cerca 
de $10 \mathrm{~min}$ ) ou pela introduçáo de gravetos. Após a saída dos indivíduos dos olheiros de cada ninho, foram realizadas coleta de espécimes para montagem e identificação. $\mathrm{O}$ segundo inventário consistiu em checar a atividade de todas as colônias ativas mapeadas em 2005 e registrar a presença das novas colônias.

A fim de testar se o número de sementes removidas e a desfolha de plântulas por saúvas são maiores na parcela tratamento do que na parcela controle, foram realizados dois tipos de experimentos entre julho - dezembro de 2007: 1) 48 horas de observaçóes e 2) transecção.

Experimento 48 horas de observação: Em cada parcela foram estabelecidas seis estaçôes experimentais: três na borda e três no interior da floresta (distante $500 \mathrm{~m}$ da borda e paralelo à mesma). Em cada estação, foram instalados pontos amostrais (distantes $10 \mathrm{~m}$ entre si) para observação de remoção e desfolha de plântulas.

Para a observação da remoção de sementes, foram estabelecidos três círculos no cháo da floresta $(40 \mathrm{~cm}$ de diâmetro) em cada ponto. Cada círculo continha sementes de 10 espécies de plantas (Tabela 1) aleatoriamente distribuídas em pequenos montes. Cada monte continha 10 sementes da mesma espécie identificada por um numeral escrito em um palito de picolé enterrado no solo. A serapilheira de dentro dos círculos foi removida durante a instalação do experimento a fim de facilitar a visualização e contagem das sementes deixadas. Nos mesmos pontos, ao lado dos círculos, foi transplantadas dez pares de plântulas de Protium sp. (Burceraceae), uma

Tabela 1 - Espécies das plantas cujas sementes foram utilizadas nos experimentos de remoção por saúvas em floresta de transição AmazôniaCerrado.

\begin{tabular}{lccc}
\hline \multirow{2}{*}{ Espécie de planta } & Peso & \multicolumn{2}{c}{ Experimento } \\
\cline { 2 - 4 } & $(\mathrm{g})$ & 48 horas & Transecção \\
\hline Pera arborea mutis (Euphorbiaceae) & 0,042 & $\mathrm{x}$ & \\
Schefflera morototonii (Araliaceae) & 0,047 & $\mathrm{x}$ & \\
$\begin{array}{l}\text { Enterolobium schomburgkii } \\
\text { (Mimosaceae) }\end{array}$ & 0,058 & $\mathrm{x}$ & \\
Guatteria sp. (Annonaceae) & 0,183 & $\mathrm{x}$ & \\
\hline Solanum lycocarpum (Solenaceae) & 0,002 & $\mathrm{x}$ & \\
$\begin{array}{l}\text { Sclerolobium paniculatum } \\
\text { (Caesalpinoidae) }\end{array}$ & 0,049 & $\mathrm{x}$ & \\
\hline Ormosia paraensis (Fabaceae) & 0,456 & $\mathrm{x}$ & \\
\hline Byrsomina aerugo (Malpighiaceae) & 0,495 & $\mathrm{x}$ & \\
\hline Terminalia amazonica (Combretaceae) & 0,509 & $\mathrm{x}$ & \\
\hline Brachiaria decumbens (Poaceae) & 0,002 & $\mathrm{x}$ & \\
\hline Mabea sp. (Euphorbiaceae) & 0,09 & & $\mathrm{x}$ \\
\hline Copaifera langsdorffii (Caesalpinioideae) & 0,47 & & $\mathrm{x}$ \\
\hline Myrcia sp. (Myrtaceae) & 0,08 & & $\mathrm{x}$ \\
\hline
\end{tabular}

espécie comum na área de estudo. Cada par consistiu de uma planta protegida individualmente com tubo de PVC e outra planta sem esta proteção, totalizando 60 pares (30 na borda e 30 no interior). Cada estaçóes experimental foi observadas por 48 horas ininterruptamente. O tipo e o número de sementes removidas por círculo e de folhas removidas por plântula foram registrados. Para as observaçôes noturnas, foram utilizadas luzes artificiais.

Experimento de transecção: Um transecto de $500 \mathrm{~m}$ de comprimento (paralelo à borda da floresta) foi estabelecido em cada parcela. A cada $10 \mathrm{~m}$ deste transecto foram instaladas estaçôes experimentais de observação de remoção de sementes, como descrito anteriormente, de sete espécies de plantas (Tabela 1). Nas locais foram oferecidos galhos recém cortados de manga (Mangifera indica: Anacardiaceae), uma espécie exótica comumente consumida pelas saúvas (obs. pessoal) e disponível na área de estudo. As observaçôes de sementes começavam às 8 horas e checadas a cada $2-3$ horas até às 18:30 - 19:00 período ainda possível de localização e contagem das sementes sob luz natural. Foram consideradas sementes removidas por saúva, somente aquelas que foram observadas sendo carregadas por essas formigas.

Em cada estação, também foram transplantados três galhos (separadas por $50 \mathrm{~cm}$ ) no chão da floresta. Os galhos foram colocados ao anoitecer e observados até o seu descobrimento e começo da desfolha por saúvas. Ao amanhecer do dia seguinte, todos os galhos foram vistoriados nas duas parcelas.

A presença do fogo como agente causador do aumento da abundância de colônias de saúva e do aumento da intensidade de suas atividades (remoçâo de sementes e desfolha de plântulas) foi testada por meio de análises de variância (ANOVA).

\section{RESULTADOS E DISCUSSÃO}

\section{Abundância de ninhos de saúva}

O inventário realizado em 2005 nas duas parcelas revelou, respectivamente, 122 colônias $(2,4$ ninhos/ha) e 71 (1,4 ninhos/ha). O número médio de ninhos por transecto de um km foi $12,2( \pm 4,8)$ na parcela tratamento e $7,9( \pm 3,6)$ na parcela controle. As espécies registradas foram: Atta sexdens, $A$. cephalotes, A. laevigata, sendo essas duas últimas residentes de ninhos maduros (murundu formado com área superficial igual ou superior a $15 \mathrm{~m}^{2}$ ) e de baixa freqüência. Na parcela controle, apenas um ninho maduro foi registrado (A. cephalotes) e na parcela tratamento, aproximadamente $5 \%$ das colônias eram maduras (cinco ninhos de $A$. cephalotes e um ninho de $A$ laevigatta). A espécie A. sexdens, representou mais de $90 \%$ das colônias (Tabela 2) e apresentou ninhos com o murundu em formação ou já formado, mais ainda inconsistente (terra ainda náo compactada). O número de colônias dessa espécie 
Tabela 2 - Abundância de colônias de Atta sexdens em 2005 - 2007 por parcela de floresta de transição Amazônia-Cerrado.

\begin{tabular}{ccccccc}
\hline \multirow{2}{*}{ Parcela } & \multicolumn{2}{c}{ Colônia Ativa } & \multicolumn{2}{c}{ Colônia Abandonada } & \multicolumn{2}{c}{ Colônia Nova } \\
\cline { 2 - 8 } & Controle & Fogo & Controle & Fogo & Controle & Fogo \\
\hline Ano 2005 & 65 & 110 & & & & \\
Ano 2007 & 75 & 150 & 18 & 27 & 28 & 67 \\
\hline
\end{tabular}

teve um aumento significativo apenas na parcela perturbada tratamento $(\mathrm{F}=8,249 ; \mathrm{df}=1 ; p=0,005 ; \mathrm{n}=32)$. O tempo $(\mathrm{F}$ $=0,079 ; \mathrm{df}=1 ; p=0,780 ; \mathrm{n}=32)$ ou sua interação com as parcelas $(\mathrm{F}=2,054 ; \mathrm{df}=1 ; p=0,163 ; \mathrm{n}=32)$ não mostrou efeito no aumento da abundância de suas colônias. $\mathrm{O}$ número de ninhos de variou em relação à distância da borda da floresta (Figura 1) mostrando uma diminuição significativa com o aumento da sua distância nas duas parcelas (Figura 2).

Esses resultados corroboram com diversos estudos que mostram aumento das colônias de saúvas em áreas perturbadas (Fowler 1983; Jaffé e Vilela 1989; Vasconcelos e Cherret 1995, 1998; Barbosa et al. 2005; Vasconcelos et al. 2006; Urbas et al. 2007; Wirth et al. 2007; Meyer et al. 2009). Em um estudo pioneiro na Amazônia Central, Vasconcelos e Cherrett (1998) apontam Atta sexdens e A. laevigata como espécies favorecidas pela conversão da floresta primária em floresta secundária.

Em um cenário de perturbaçóes de fogo anual, as populaçóes de saúva tendem a aumentar, seguido uma tendência comum dos herbívoros, repetidamente mais observados nas bordas (Barbosa et al. 2005; Wirth et al. 2007). Um estudo de Urbas et al. (2007) mostrou que a herbivoria aumentou consideravelmente nas bordas em conseqüência da redução da área de forrageamento por colônia e do índice de área foliar nesse local. Provavelmente são as modificaçôes estruturais da vegetação causada pelo fogo (ex: maior

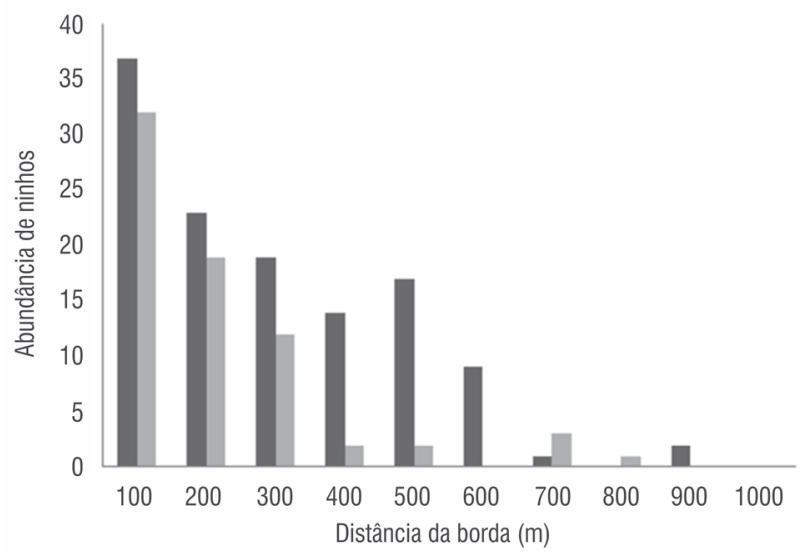

Figura 1 - Abundância de ninhos de Atta spp. por classe de distância da borda em duas parcelas de floresta de transição Amazônia-Cerrado. Barras pretas: tratamento; cinzas: controle. abertura de dossel ou sub-bosque) que estão determinando à proliferação de saúvas nesse ambiente, assim como nas bordas da parcela não queimada. A maior disponibilidade de luz nesses locais pode: 1) promover o surgimento de plantas pioneiras, que são mais palatáveis aos herbívoros; 2) atrair a presença de rainhas fundadoras (ainda em pleno vôo nupcial) e 3) favorecer a construção e permanência do murundu inicial (Barbosa et al. 2005; Vasconcelos et al. 2006; Urbas et al. 2007; Wirth et al. 2007; Meyer et al. 2009).

Para Moutinho et al. (2003), a magnitude dos efeitos de $A$. sexdens sobre a vegetação, depende da densidade e do tamanho dos ninhos. No presente estudo, embora a densidade de ninhos dessa espécie tenha sido alta, não foram registradas colônias residindo em ninhos maduros. Esses ninhos, segundo Holldobler e Wilson (1990) são caracterizados por: grande quantidade de indivíduos (até três milhóes) e cavidades subterrâneas (centenas), alta profundidade (até $7 \mathrm{~m}$ de acordo com Moreira et al. 2004) e murundu ocupando área com até mais de $50 \mathrm{~m}^{2}$.

Uma maior densidade de ninhos maduros significa maior número de operárias em atividade. Estas atividades de acordo com Moutinho et al. (2003), podem ser contrabalanceadas pelos benefícios dos ninhos sobre o solo: (alteraçóes físicoquímicas como: redução da resistência do solo à penetração de raízes e aumento da fertilidade) e sobre a vegetaçáo (aumento na concentração foliar de Cálcio: Sternberg et al. 2006 e Fósforo: Carvalho 2008 e no crescimento em diâmetro do caule: Carvalho 2008).

$\mathrm{Na}$ floresta de transição estudada, a densidade de ninhos maduros foi naturalmente baixa e o fogo está promovendo o surgimento de novas colônias. Portanto, é improvável que os ninhos favoreçam a recuperação da vegetação pós-fogo nessa fase. Provavelmente a médio e longo prazo, o avanço da sucessão florestal reduza a proliferação de ninhos de Atta spp. e a assim, a vegetação possa beneficiar-se dos ninhos de saúva.

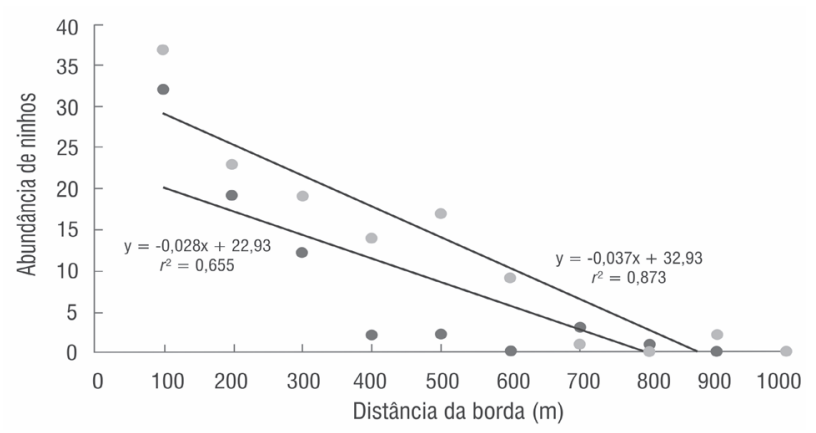

Figura 2 - Relação entre abundância de ninhos de saúva Atta spp. por transecto de $200 \mathrm{~m}$ e distância da borda em duas parcela de floresta de transição Amazônia-Cerrado. Pontos pretos: controle; cinza: tratamento. 


\section{Remoção de sementes por saúvas}

$\mathrm{Na}$ parcela de fogo, o número de sementes removidas na borda foi $3,10( \pm 3,15)$ e no interior $4,0( \pm 3,46)$; Figura. $3 . \mathrm{Na}$ parcela controle, as bordas revelaram em média 4,3 $( \pm 2,73)$ sementes removidas enquanto que no interior a média reduziu para $1,26( \pm 2,22)$. Não houve efeito da parcela $(\mathrm{F}=2,709$; $\mathrm{gl}=1 ; p=0,104)$ nem do local $(\mathrm{F}=5,245 ; \mathrm{gl}=1 ; p=0,025)$ mas sim um efeito significativo da interaçáo entre esses dois fatores ( $\mathrm{F}=17,828 ; \mathrm{gl}=1 ; p=0,001)$ e da espécie de semente $(\mathrm{F}=6,866 ; \mathrm{gl}=9 ; p=0,001)$. O teste de "post hoc" mostrou que a remoção foi maior no interior da parcela tratamento que no interior da parcela controle (Tukey $=-2,733 ; p=0,001$ ) e que esta teve mais remoçáo nas bordas (Tukey $=3,033 ; p$ $=0,001)$ que no interior. A parcela tratamento náo revelou diferenças entre esses dois locais (Tukey $=0,900 ; p=0,524$ ). A transecção mostrou remoção significativamente maior na parcela tratamento $(2,4 \pm 2,9)$ em comparação a controle $(1,8$ $\pm 2,4 ; t=-3.202 ; p=0,001 ; \mathrm{n}=430)$. A espécie mais removida foi Pera arborea mutis. O peso médio de sua semente $(0,042 \mathrm{~g})$ mostrou um efeito significativo sobre o número de sementes removidas no experimento de 48 horas $\left(r^{2}=0,339 ; p=0,000\right)$ e no experimento de transecção $\left(r^{2}=0,297 ; p=0,000\right)$.

Apesar da predação, as saúvas podem atuar como dispersor secundário de sementes e promover a germinação de diversas espécies florestais (Farii-Brener e Silva 1995; Oliveira et al. 1995; Pizzo e Oliveira 1998; Leal e Oliveira 2000), pois ao manipular as sementes, essas formigas diminuem o ataque

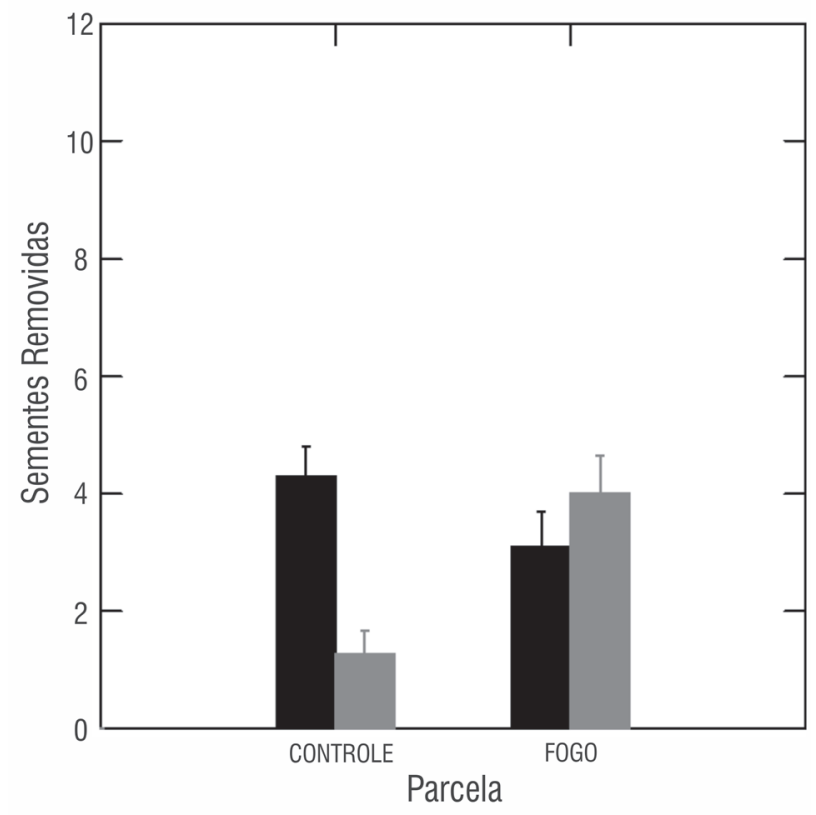

Figura 3 - Número médio de sementes ( \pm erro padrão) removidas por saúvas em parcelas de 50 ha em floresta de transição Amazônia - Cerrado. Barras pretas: borda; cinzas: interior. de fungos patogênicos, devido à ação fungicida e bactericida de sua glândula metapleural (Farji-Brener e Silva 1995; Oliveira et al. 1995). Apesar disso, semente removida não significa dispersada, principalmente porque as saúvas podem consumi-las ou torná-la inviável por meio de descarte em altas profundidades. Escavaçóes de um formigueiro de $A$. sexdens (Nepstad 1998) revelaram sementes viáveis enterradas à 0,5 $\mathrm{m}$ da superfície do solo.

Mesmo que as sementes sejam descartadas em solo sob a influência dos ninhos e, portanto, rico em nutrientes, isso seria um benefício também para plantas exóticas como demonstrado por Farji-Brener e Ghermandi (2008). Os estudos de Moutinho et al. (2003), mostram que o tipo de estágio em que a vegetação se encontra (inicial ou avançado) influência o papel ecológico das saúvas. Como citado na seção anterior, o avanço da vegetação tende a reduzir o número de ninhos estabelecendo-se no ambiente e conseqüentemente a diminuir as pressôes das saúvas sobre a vegetação.

Outro aspecto importante na caracterizaçáo do papel das saúvas está relacionado à sua seletividade. Uma predação seletiva e intensa de propágulos sobre uma espécie (como aquela observada para a espécie $P$. arborea mutis) pode resultar em vantagem para outra, desde que esta cresça e acumule fitomassa de forma rápida. Desta forma, a predação influenciaria no aumento da velocidade da regeneração florestal e não no seu retardo ou redução. Em um cenário assim, a influência mais marcante das saúvas, provavelmente seria em modificar a composição da vegetação original.

\section{Desfolha de plântulas por saúvas}

Os transplantes de Protium sp. não apresentaram desfolha total, mas sofreram cortes de folhas inteiras (por meio do pecíolo). Enquanto mais de duas folhas $(2,1 \pm 3,3)$ foram arrancadas por planta transplantada na parcela tratamento, aproximadamente uma $(0,8 \pm 1,5)$ foi registrada na parcela controle. Somente a parcela mostrou efeito significativo ( $F$ $=14.983 ; \mathrm{gl}=1 ; p=0,001) . \mathrm{O}$ local $(\mathrm{F}=5.257 ; \mathrm{gl}=1 ; p$ $=0,023)$ e a proteçáo de cano de $\operatorname{PVC}(1,975 ; \mathrm{gl}=1 ; p=$ $0,161)$ não influenciaram a abundância de folhas removidas. Aproximadamente 76\% (50 indivíduos) dos galhos de Mangifera indica foram completamente desfolhados na parcela tratamento. $\mathrm{Na}$ parcela controle, a porcentagem praticamente caiu pela metade ( $37 \%$ ou 24 plântulas; Figura 4). A abundância média de galhos desfolhados foi $0,7( \pm 0,4)$ na parcela tratamento e $0,3( \pm 0,4)$ na parcela controle.

Embora a resistência das plantas ao ataque das saúvas varie de acordo com a espécie, a desfolha total, como constatada para os galhos de manga, conduz a altas taxas de mortalidade, segundo Vasconcelos e Cherrett (1998). Algumas espécies não resistem a ataques repetidos nas primeiras semanas após a germinação. 


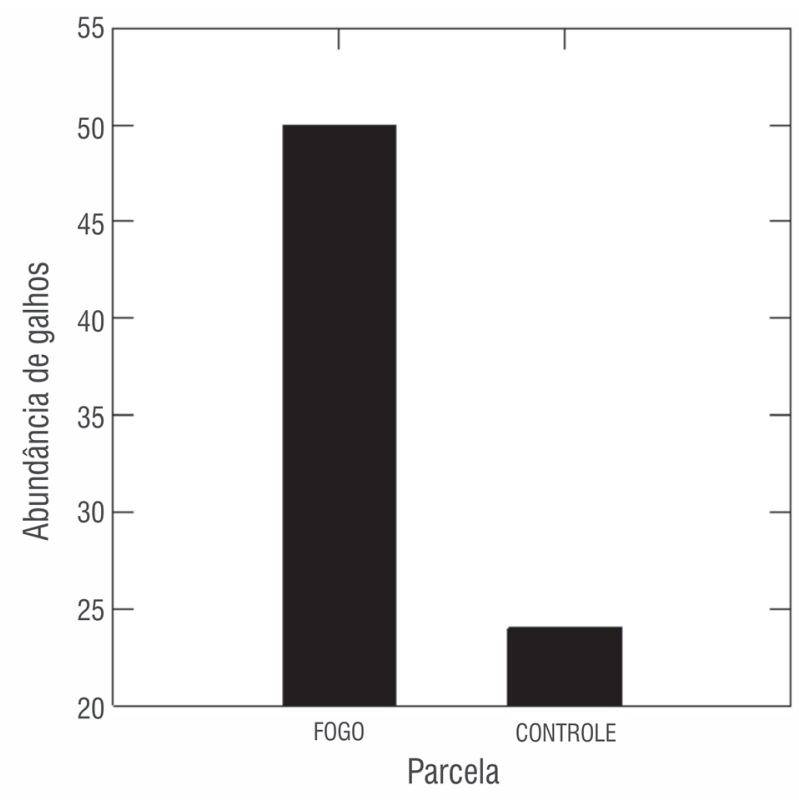

Figura 4 - Número total de galhos de Mangifera indica completamente desfolhado por saúva em duas parcelas de floresta de transição Amazônia - Cerrado.

As saúvas são consideradas o herbívoro mais importante da regiấo Neotropical devido à grande quantidade de folhas que cortam para o cultivo de um fungo simbionte (Holdobler e Wilson 1990). Uma colônia de Atta pode consumir entre 51 a $500 \mathrm{~kg}$ de plantas por ano em um ambiente natural segundo estimativa de Costa et al. 2008. A herbivoria, no entanto, é direcionada por sua alta seletividade, ilustrada nesse estudo, pelo ataque sutil aos transplantes de Protium sp. em comparação a forte pressão sobre as folhas de manga. Um estudo recente de Mundim et al. (2009) mostra que as folhas cortadas por A. laeviatta tiveram maior concentração de vários macronutrintes (Nitrogênio, Fósforo e Potássio) e de alguns micronutrientes (Zinco e Cobre) e que houve uma tendência de correlaçáo entre esses elementos. Desta forma foi difícil determinar qual (ou quais) o nutriente mais importante na seleção das saúvas. Embora seja comum a qualidade nutricional das folha influenciar a escolha dos herbívoros de um modo geral (Huberty \& Denno 2006), a preferência das saúvas parece mais relacionada ao crescimento do substrato do seu jardim de fungo.

Embora diversos e intricados fatores possam influenciar a seletividade da planta hospedeira, o fato é que ao selecionar espécies, as saúvas podem favorecer a permanência de gramíneas e arbustos, modificando a composição das comunidades e atuando como barreira à sucessão vegetal pós-fogo. Esse papel pode modificar-se quando a pressão seletiva resulta em aceleração (e não retardo ou diminuição) na velocidade de recomposiçáa da vegetaçáo. Segundo Vasconcelos e Cherrett (1997) as chances de uma plântula ser atacada está intimamente relacionada com os estágios inicias de regeneração florestal e declina com o tempo.

Nesse contexto, seriam precisos mais estudos para estabelecer um balanço dos efeitos positivos e negativos das saúvas na recuperação da floresta de transição amazônica recuperando-se do fogo. Como o abandono de terras é um evento freqüente na Amazônia (cerca de 200 a $300 \mathrm{mil} \mathrm{km}{ }^{2}$ de acordo com Zarin et al. 2001) é de extrema importância entender o papel das saúvas para os processos de regeneraçáo florestal.

\section{CONCLUSÕES}

Embora seja prematuro estabelecer um balanço preciso entre os efeitos negativos e positivos das saúvas no processo de regeneração florestal pós-fogo, este estudo permite estabelecer algumas previsōes. Os efeitos negativos (ex: predação de sementes e herbivoria) devem predominar sobre os positivos (aumento da fertilidade do solo e crescimento da vegetação circundante), se confrontados nos estágios iniciais de sucessão. Pois, a abundância de colônias nessa fase é mais alta (a atividade de corte é bastante intensa causando grande impacto sobre o estabelecimento da vegetação) e os ninhos ainda não apresentam tamanhos e/ou profundidade suficiente para promover efeitos significativos no solo e conseqüentemente sobre a vegetação.

Com o tempo e avanço da vegetação a situação tende a inverter-se. As colônias estabelecidas residirão em ninhos maduros e as formigas diminuirão suas atividades, já que a estrutura da floresta mais complexa tornar-se-á inadequada para sua sobrevivência. Nessa fase, com o declínio da atividade de corte, os ninhos apresentam efeitos mais relevantes e podem beneficiar o processo de regeneraçáo florestal.

\section{AGRADECIMENTOS}

Ao Programa Bolsa de Estudos para a Conservação da Amazônica (BECA) do Instituto Internacional de Educação no Brasil (IEB) e a Fundaçáo de Amparo a Pesquisa do Estado da Bahia (FAPESB) pelo suporte financeiro. Ao Instituto de Pesquisa Ambiental da Amazônia (IPAM) pelo apoio logístico, especialmente a equipe técnica: Osvaldo Portela, Darlisson Nunes, Raimundo Mota, Roberto Baena, Joelson Serrão, Aderlan Pedroso, Adriano Portela e Artemísia.

\section{BIBLIOGRAFIA CITADA}

Araújo, M.S.; Della-Lucia, M.T.C.; Picanço, M.C. 2004. Impact of burning trash in cane sugar in the daily rhythm of foraging of Atta bisphaerica Forel (Hymenoptera, Formicidae). Revista Brasileira de Zoologia, 21: 33-38 (in Portuguese). 
Balch, J.K.; Nepstad, D.; Brando, P.M.; Curran, L.M.; Portela, O.; Carvalho Jr, O.; Lefebvre, P. 2008. Negative fire feedback in a transitional forest of southeastern Amazonia. Global Change Biology, 14: 2276-2287.

Barbosa, V.S.; Leal, I. R.; Iannuzzi, L.; Almeida-Cortez, J. 2005. Distribution pattern of herbivorous insects in a remnant of Brazilian Atlantic forest. Neotropical Entomological, 34: 1-11.

Barlow, J.; Peres, C. A. 2003. Low-intensity fire, new threat in the Amazon. Ciência Hoje, 34(199): 24-29 (in Portuguese).

Barlow, J.; Peres, C. A.; Logan, B. O.; Haugaasen, T. 2003. Large tree mortality and the decline of forest biomass following Amazonian wildfires. Ecolology Letters, 6: 6-8

Buckley, R. 1982. Ant-plant interactions in Australia. The Hangue. Dr. W. Junk Publisher, 162 pp.

Carvalho, KS. 2008. Influences of the leafcutter ant nests (Formicidae: Attini) in nutrition, growth and protection of vegetation fire in Amazonian transitional forest. Tese de Doutorado, Museu Paraense Emilio Goeldi, Belém, PA, Brasil. 145 pp (in Portuguese).

Cochrane, M. A.; Alencar, A.; Schulze, M. D.; Souza, C. M.; Nepstad, D.; Lefebvre, P.; Davidson, E. A. 1999. Positive feedbacks in the fire dynamic of closed canopy tropical forests. Science, 284: 1832-1835.

Cochrane, M. A; Laurence, W. F. 2002. Fire as large-scale edge effect in Amazonian forest. Journal Tropical Ecology, 18: 311-325.

Costa, A.N.; Vasconcelos, H.L; Viera-Neto, F.H.M; Bruna, E.M. 2008. Do herbivores exert top-down effects in Neotropical savannas? Estimates of biomass consumption by leaf-cutter ants. Journal of Vegetation Science, 19: 849-854.

Curran, L. M., Caniago, I.; Paoli, G. D.; Astianti, D.; Kusneti, M.; Leighton, M.; Nirarita, C. E. 1999. Impacto f El Nino and logging on canopy tree recruitment in Borneo. Science, 286: 2184-2188.

Farji-Brener, A.G.; Silva, J. 1995. Leaf-cutting ants and soil fertility in a well-drained savanna in western Venezuela. Biotropica, 27: 250-253.

Farji-Brener, A.G.; Medina, C. 2000. The importance of where to dump the refuse: seed banks and fine roots in nest of the leaf-cutting ants Atta cephalotes and A. colombica. Biotropica, 32 (1): 120-126.

Farji-Brener, A.G. 2000. Leaf-cutting ant nest in temperate environments: mounds, mound damage and nest mortality rate in Acromyrmex lombicornis. Stud. Neotropical Fauna \& Environment, 35: 131-138.

Farji-Brener, A.G.; Illes, A. 2000. Do leaf-cutting ant nests make "bottom-up" in neotropical rain forest? : a critical review of the evidence. Ecology Letters, 3: 219-227.

Farji-Brener, A.G. 2001. Why are leaf-cutting ants more common in early secondary forest than in old-growth tropical forest? An evalution of the palatable forage hypothesis. Oikos, 92: 169-170.

Farji-Brener, A.G. 2005. The effect of abandoned leaf-cutting ant nests on plant assemblage composition in a tropical rainforest of Costa Rica. Écoscience, 12 (4): 554-560.
Farji-Brener, A.G.; Ghermandi, L. 2008. Leaf-cutting ant nests near roads increase fitness of exotic plant species in natural protected areas. Proceedings of the Royal Society, 275: 1431-1440.

Fowler, H.G. 1983. Distribution patterns of Paraguayan leafcutting ants (Atta and Acromyrmex) (Formicidae: Attini). Studies Neotropical Fauna \& Environment, 18: 121-138.

Garrettson, M.; Stetzel, J.; Halpern, B.; Hearn, J.; Lucey, B.; McKone, M. 1998. Diversity and abundance of understory plants on active and abandoned nest of leaf-cutting ants (Atta cephalotes) in a Costa Rica rain forest. Journal of Tropical Ecolgy, 14: 17-26.

Haugaasen, T., Barlow, J.; Peres, C. A. 2003. Surface wildfires in Central Amazonia: short-term impact on the forest structure and carbon loss. Forest Ecology and Management, 179: 321-331.

Huberty, A.F.; Denno, R.F. 2006. Consequences of nitrogen and phosphorus limitation for the performance of two planthoppers with divergent life-history strategies. Oecologia 149: 149-444.

Jaffé, K.; Vilela, E. 1989. On nest densites of the leaf-cutting ant Atta cephalotes in tropical primary forest. Biotropica, 21: 234-236.

Leal, I.R.; Oliveira, P.S. 2000. Foraging ecology of attine ants in a Neotropical savanna: seasonal use of fungal substrate in the cerrado vegetation of Brazil. Insectes Sociaux, 47: 376-382.

Majer, J.D. 1989. Animals in primary sucession. The role of fauna in reclaimed lands. Cambridge. $547 \mathrm{pp}$.

Meggers, B. J. 1994. Archeological evidence for the impact of Mega-Nin־o events of Amazonia during the past two millennia. Climatic Change, 28:321-338.

Meyer S.T.; Leal I.R.; Wirth, R. 2009. Persisting hyper-abundance of leaf-cutting ants (Atta spp.) at the edge of an old Atlantic forest fragment. Biotropica, 41 (in press)

Moore, P.D. 1996. Fire damage soils our forests. Nature, 384: 312-313.

Moreira, A. A.; Forti, L. C.; Boaretto, M. A. C.; Andrade, A. P. P.; Lopes, J. F. S.; Ramos, V. M. 2004. External and internal structure of Atta bisphaerica Forel (Hymenoptera, Formicidae) nest . Journal of Applied Entomology, 128: 200-203.

Moutinho, P. 1995. Stop the ants are not. Ciência Hoje, 18 (106): 10-11. (in Portuguese).

Moutinho, P. 1998. Impacts of Grazing on the Formation of the Ant Fauna: Consequences for Forest Restoration in the Eastern Amazon, p. 155-170. In: Gascon, C.; Moutinho, P. (Eds). Dinâmica, Recuperaçâo e Manejo da Floresta Amazônica. Instituto Nacional de Pesquisas da Amazônia, Manaus, Amazonas. (in Portuguese).

Mundim, F.M.; Costa, A.N; Vasconcelos, H.L. 2009. Leaf nutrient content and host plant selection by leaf-cutter ant Atta laevigatta in a Neotropical savanna. Entomologia Experimentalis et Applicata, 130(1): 47-54.

Nepstad, D.C.; Uhl, C. Pereira, C.A.; Silva, J.M.C. 1996.A comparative study of tree establishment in abandoned pasture and mature forest of eastern Amazonia. Oikos, 76: 25-39.

Nepstad, D.C.; 1998. Barriers to establishment of trees in abandoned pastures in the Amazon: the seed bank, seed predation, herbivory 
and drought, p. 191-218. In: Gascon, C.; Moutinho, P. (Eds). Dinâmica, Recuperação e Manejo da Floresta Amazônica.Instituto Nacional de Pesquisas da Amazônia, Manaus, Amazonas. (in Portuguese).

Nepstad, D.; Veríssimo, A.; Alencar, A.; Schlesinger, L. P., P.; Moutinho, P.; Mendoza, E.; Cochrane, M. A.; Brooks, V. 1999. Large-scale impoverishment of Amazonian forest by logging and fire. Nature, 398: 505-508.

Oliveira, P.S.; Galetti, M.; Pedroni, F.; Morellato, L.P.C. 1995. Seed cleaning by Mycocepurus goeldii ants (Attini) facilitates germination in Hymenea courbaril (Caesalpiniaceae). Biotropica, 27: $518-522$.

Oren, D. 2005. Portfolio of important areas for biodiversity conservation in Brazil: an analise by ecorregion. The Nature Conservancy, Brasília, DF. 36 pp. (in Portuguese).

Pizzo, M.A.; Oliveira, P.S. 1998. Interaction between ants and seeds of a nonmyrmecochorous neotropical tree, Cabrelea canjerana (Meliaceae), in the Atlantic forest of Southeast Brazil. American Journal of Botany, 85: 669-674.

Rao, M. 2000. Variation in leaf-cutter ant (Atta sp.) densities in forest isolates: the potential role of predation. Journal of Tropical Ecology 16: 209-225.

Roces, F.; Kleineidam, C. 2000. Humidity preference for fungus culturing by workers of the leaf-cutting ant Atta sexdens rubropilosa. Insectes Sociaux, 47: 348-350.

Saldarriaga, J.G; West, D. C.; Tharp, M. L.; Ulh, C. 1988. Longterm chronosequence of forest sucession in the upper Rio Negro of Colombia and Venezuela. Journal of Ecology, 76: 938-958.

Santos, B. A., Peres, C. A.; Oliveira, M. A.; Grillo, A.; Alves-Costa, C. P.; Tabarelli, M. 2008. Drastic erosion in functional attributes of tree assemblages in Atlantic forest fragments of northeastern Brazil. Biological Conservation, 141: 249-260.
Siegert, F.G.; Rueckre, G.; Hinrichs, A.; Hoffmann, A. A. 2001. Increased damage from fires in logged forests during drougths caused by El Niño. Nature, 414: 437-440.

Uhl, C.; Kauffman, J. B. 1990. Deforestation effects on fire susceptibility and the potential response of tree species to fire in the rain forest of the eastern Amazon. Ecology, 71: 437-449.

Urbas, P.; Araujo Jr, M.V.; Leal, I. R.; Wirth, R. 2007. Cutting More from Cut Forest: Edge Effects on Foraging and Herbivory of Leaf-Cutting Ants in Brazil. Biotropica, 39 (4): 489-495.

Vasconcelos, H.L,; Cherrett, J.M. 1995. Changes in leaf-cutting ant populations (Formicidae: Attini) after the clearing of mature forest in Brazilian Amazonia. Stud. Neotropical Fauna \& Environment, 30: 107-113.

Vasconcelos, H.L.; Cherrett, J.M. 1997. Leaf-cutting ants and early forest regeneration in central Amazonia: Effects of herbivory on tree seedling establishment. Journal Tropical Ecology, 13:357-370.

Vasconcelos, H.L. e Cherrett, J.M. 1998. Effects of Herbivory by Atta laevigata (Fr. Smith) on the Regeneration of Woody Plants in em Area Abandoned Agricultural Area of Central Amazonia, p. 171-178. In: Gascon, C.; Moutinho, P. (Eds). Dinâmica, Recuperação e Manejo da Floresta Amazônica. Instituto Nacional de Pequisas da Amazônia, Manaus. (in Portuguese).

Vasconcelos, H.L.; Vieira-Neto, E.H.M.; Mundim, F.M. 2006. Roads Alter the Colonization Dinamics of Keystone Herbivore in Neotropical Savannas. Biotropica, 38 (5): 661-665.

Wirth, R., Meyer, S.T.; Almeida, W.R.; Araujo Jr, M.V.; Barbosa, V.S.; Leal, I. R. 2007. Increasing densities of leaf-cutting ants (Atta spp.) with proximity to the edge in a Brazilian Atlantic forest. Jornal of Tropical Ecology, 23: 501-505.

Zarin, D.J; Ducey, M.J.; Tucker, J.M.; Salas, W.A. 2001. Potential biomass accumulation in Amazonian regrowth forest. Ecosystems, 4: 658-668.

Recebido em 29/11/2010

Aceito em 18/02/2011 